\title{
Lipophagy: A New Perspective of Natural Products in Type 2 Diabetes Mellitus Treatment
}

\author{
Mingyue Huang ${ }^{1,2, *}$ \\ Xinyu Yang ${ }^{2, *}$ \\ Zhenzhen Wang ${ }^{3}$ \\ Jianglan Long ${ }^{3}$ \\ Aiting Wang ${ }^{3}$ \\ Yi Zhang ${ }^{4}$ \\ Dan $\mathrm{Yan}^{3}$

\begin{abstract}
'College of Pharmacy, Chengdu University of Traditional Chinese Medicine, Chengdu, 6III30, People's Republic of China; ${ }^{2}$ Beijing Key for Evaluation of Rational Drug Use, Beijing Shijitan Hospital, Capital Medical University, Beijing, 100038, People's Republic of China; ${ }^{3}$ Department of Pharmacy, Beijing Friendship Hospital, Capital Medical University, Beijing, I00050, People's Republic of China; ${ }^{4}$ Department of Traditional Chinese Medicine and Natural Medicine, Chongqing Institute for Food and Drug Control, Chongqing, 40II2I, People's Republic of China

*These authors contributed equally to this work
\end{abstract} \\ Laboratory of Bio-Characteristic Profiling
}

\footnotetext{
Correspondence: Dan Yan Department of Pharmacy, Beijing Friendship Hospital, Capital Medical University, No. 95, Yong'an Road, Xicheng District, Beijing, 100050, People's Republic of China

Tel +86 10-63139318

Email pharmsci@126.com

Yi Zhang

Department of Traditional Chinese Medicine and Natural Medicine, Chongqing Institute for Food and Drug Control, No. I, Chunlan 2nd Road, Yubei District, Chongqing, 40II2I, People's Republic of China

Tel +86 23-8607277l

Email zhangyi@cqifdc.org.cn
}

\begin{abstract}
Autophagy has been reported to involve in the pathogenesis of type 2 diabetes mellitus (T2DM), which protects the insulin target tissues and pancreatic $\beta$-cells. However, autophagy is inhibited when the cells are lipid overload. That, in turn, increases the accumulation of fat. Lipotoxicity caused by excessive lipid accumulation contributes to pathogenesis of T2DM. Therefore, it is undeniable to break the vicious circles between lipid excess and autophagy deficiency. Lipophagy, a selective form of autophagy, is characterized by selective breakdown of lipid droplets (LDs). The nutritional status of cells contributes to the way of autophagy (selective or non-selective), while selective autophagy helps to accurately remove excess substances. It seems that lipophagy could be an effective means to decrease abnormal lipid accumulation that leads to insulin resistance and $\beta$-cell impairment by removing ectopic LDs. Based on this process, many natural compounds have been reported to decrease lipid accumulation in tissues through autophagy-lysosomal pathway, which gradually highlights the significance of lipophagy. In this review, we focus on the mechanisms that lipophagy improves T2DM and natural products that are applied to induce lipophagy. It is also suggested that natural herbs with rich contents of natural products inducing lipophagy would be potential candidates for alleviating T2DM.
\end{abstract}

Keywords: lipophagy, lipid metabolism, type 2 diabetes mellitus, autophagy, natural products

\section{Introduction}

Type 2 diabetes mellitus (T2DM) is a complex metabolic disease with a high incidence worldwide. Over the past decades, many approaches have been adopted to improve T2DM including lifestyle intervention by weight loss via exercise and diet, agents to insulin sensitization as well as multitasking agents that enhance efficiency of glucosestimulated insulin secretion and insulin-stimulated glucose uptake. ${ }^{1}$ It still remains unclear about its pathogenesis and effective therapeutic approaches, although much work has been accomplished. Among them, visceral fat is highly associated with T2DM, which shows a greater propensity for overweight and obesity. ${ }^{2,3}$ Excessive fat accumulation is prone to occur in liver, which leads to increased lipogenesis and gluconeogenesis and decreased glycogen synthesis. ${ }^{4}$ Meanwhile, evidence also suggests that excessive fat accumulation is stored in muscle, pancreas, etc, ${ }^{2,5}$ which is of great importance in driving the pathogenesis and progression of T2DM. Thus, it is conducive to improve insulin resistance and restore the function of $\beta$-cells by regulating lipid metabolism and reducing abnormal fat accumulation.

Autophagy acts as a pivotal part in maintaining cellular physiology and homeostasis. ${ }^{6,7}$ It has been known to regulate the functions of pancreatic $\beta$-cells 
and insulin target tissues. Lipid accumulation is significantly increased in peripheral tissues as well as $\beta$-cells after pharmacological and/or genetic inhibition of autophagy, ${ }^{8}$ which means that autophagy is a vital part in lipid metabolism and provides new insights into improving lipid and lipoprotein abnormalities. ${ }^{9,10}$ Autophagy is characterized by widely trafficking of cytosolic structures, ranging from single protein to cell organelles. Additionally, extensive studies have reported that autophagy removes cellular components in a selective manner. Being different from the traditional non-selective autophagy, selective autophagy is designed to remove certain cargoes. These cargoes are removed or not in terms of the materials or nutrients requirement, such as mitochondria and lipid droplets (LDs). It is helpful to consider its selectivity when we try to explain this phenomenon. ${ }^{11}$ With the concept of selective autophagy being put forward, these autophagy forms including lipophagy, mitophagy, glycophagy and reticulophagy have attracted great interest because of their potential applications in diseases. ${ }^{6}$ Different mechanisms involved in the induction of selective autophagy have been elucidated in detail. ${ }^{12}$

Lipophagy is one of selective autophagy that specifically acts on degrading the LDs, ${ }^{13}$ which occurs in various cells or tissues under different conditions. It is first named because it correlates lipolysis with autophagy, which shares similarities in regulation and function. ${ }^{14}$ Emerging evidence has brought lipophagy to the area of lipid metabolism. Lipophagy process occurs generally in three conditions: normal growth, elevation in free fatty acids (FFAs), and cellular stresses. ${ }^{10,15}$ Upregulation of lipophagy occurs under states of nutrient deprivation and/or increased cellular lipid accumulation. It is supposed that lipophagy could be a desirable approach to remove the excessive lipid. However, since lipophagy was proposed, its role in lipid metabolism has prompted it to bring a new perspective for alleviating T2DM through lipid-lowering, which needs more research and attention in the industry.

Natural products are characterized by multiple properties, which have been used to improve T2DM for many years. They are applied for improving hyperglycemia, inflammation, cellular stresses and hyperlipidemia. ${ }^{16}$ In recent studies, some natural products have shown their advantages in lowering lipid through lipophagy, such as kaempferol $^{17}$ (flavonoid), and berberine ${ }^{18}$ (alkaloid). Based on consulting references, we innovatively propose that lipophagy could be a new perspective for natural products to improve T2DM, because it probably further clarifies that the regulation of lipid metabolism by autophagy actually depends on the selective autophagy termed lipophagy, which is worthy of more attention and research. This review aims at learning further information about the link between T2DM and lipophagy. At the same time, we summarize natural products that were adopted to promote lipophagy in recent literature, aiming to provide references for further studies about therapeutics by natural products for the treatment of T2DM.

\section{Disorder of Lipid Metabolism Could Cause T2DM}

Disordered lipid metabolism induces excessive lipids content and the ensuing lipotoxicity plays a major role in promoting insulin resistance and $\beta$-cell dysfunction. ${ }^{19}$ It is characterized by changes in the amount of a series of lipids, one of which is the increase in the level of triglycerides (TGs). ${ }^{20}$ These TGs are generally stored in LDs in adipocytes. Evidence shows that dyslipidemia increases T2DM risk determined by the degree of obesity and also by where fat accumulates. ${ }^{21}$ The lipid flux to non-adipose tissues increases and is subsequently deposited in non-adipose tissue when the storage capacity of adipose tissue is exceeded. ${ }^{22}$ This could be a cause of high level of visceral fat. Meanwhile, excessive lipid loading can induce an increase in lipolysis in adipocytes, resulting in the secretion of FFAs into the bloodstream. The elevated circulating FFAs result in ectopic lipid accumulating in peripheral tissues, ${ }^{23}$ which is followed by insulin resistance. Besides, long exposure to FFAs leads to $\beta$-cell lipid overload and a major negative impact on $\beta$-cell function which finally impairs insulin secretion. ${ }^{24}$ Ectopic fat accumulation in the pancreas is a response to obesity in the onset of T2DM. ${ }^{25}$ Chronic increased plasma FFAs stimulate gluconeogenesis and impairs insulin secretion in genetically predisposed individuals as well. ${ }^{26} \mathrm{It}$ is evident from the above that disordered lipid metabolism has disadvantageous influences including obesity, insulin resistance and impaired $\beta$-cells that induce the onset and progression of T2DM, which implies that maintaining lipid homeostasis and removing the ectopic fat accumulation play critical roles in improving T2DM.

\section{Lipophagy Regulates Lipid Metabolism}

\section{Abnormal Lipid Homeostasis Inhibits Autophagy}

Autophagy is a pivotal regulator of lipid metabolism and an effective means for intracellular lipids clearance. 
Recent study demonstrated that autophagy directly targets apolipoprotein B-100 to regulate very low-density lipoproteins assembly and further targets LDs. ${ }^{10}$ Generally, it is up-regulated under high fat diet (HFD), which aims to overcome variation of lipids in contents. However, damaged lipid homeostasis inhibits autophagy, which leads to a lot of adverse effects. In HFD-induced obese mice, the activation of autophagy inhibitor mTOR is increased, along with increased phosphorylation and inhibition of insulin receptor substrate 1 and impaired Akt activity. ${ }^{27}$ Moreover, in response to HFD, the mice with adenosine 5'-monophosphate (AMP)-activated protein kinase (AMPK) deficiency in adipose tissue rapidly cause glucose and insulin intolerance, and depress energy utilization in brown adipose tissue, thereby promoting the development of insulin resistance. ${ }^{28}$ High lipid loads inhibit autophagy, resulting in a further lipid accumulation and this negative feedback loop. ${ }^{29}$ With the damaged autophagy process, useful organelles and biomolecules are degraded, and the anti-apoptotic mechanism is affected, which may lead to cell death. ${ }^{6}$

\section{Lipophagy Specifically Regulates Lipid Metabolism}

FFAs from de novo synthesis and/or degradation of TGs are esterified into relatively stable neutral lipids and temporarily stored in LDs until fat mobilization for materials or nutrients requirement. Lipophagy contributes largely to fat mobilization. ${ }^{30}$ The balance of esterification and hydrolysis maintains lipid homeostasis, inhibits lipotoxicity and reduces the contents of FFAs. ${ }^{31}$ At the same time, the balance maintains normal cell physiology by supplying energy, membranes, signaling molecules, and hormones after lipids are synthesized and modified. During the process, lipophagy is selectively upregulated or downregulated in response to specific cellular needs or depending on various nutritional status. ${ }^{32}$

In addition, it is well known that autophagy is a major degradation pathway which aims to prevent the cells from death under the stress condition by removing aberrant cellular components such as mitochondria, endoplasmic reticulum (ER), and LD. ${ }^{6}$ During this process, cargoes are digested non-selectively by building a supramolecular network. ${ }^{12}$ When it comes to lipid metabolism disordered, compared with other selective autophagy, lipophagy initiation conditions, the way of recognizing and wrapping cargoes, as well as the properties and characteristics of the recognized cargoes are different. Lipophagy specifically recognizes LDs, which store most of the cellular lipids and responsible for the stability and metabolism of lipid pool. ${ }^{33}$ Lipophagy, therefore, specifically regulates lipid metabolism. These reasons also suggest that lipophagy might be an alternative way to the damage of autophagy under abnormal lipid metabolism. ${ }^{34}$ It sets up a bridge between lipid metabolism and autophagy.

\section{Lipophagy is a Conditional Autophagy}

The molecular machinery of selective autophagy is similar to the non-selective autophagy, in which the changes are same as the latter such as conversion of microtubuleassociated protein 1 light chain 3 (LC3-I), degradation of p62 and formation of autophagosomes. ${ }^{35}$ The whole process begins with the formation of phagophore assembly site, which is mediated by UNC51-like kinase complex 1 (ULK1). The first step is followed by nucleation, which requires the class III PI3K complex. Quite apart from that, there are two ubiquitin-like conjugation pathways that are recognized in the process of phagophore membrane elongation and autophagosome completion ahead of the formation of autophagosome. One of them is the ATG5ATG12 conjugate, which forms a multimeric complex with ATG16L1. And the other is the conjugation of phosphatidyl-ethanolamine to the LC3. ${ }^{36,37}$ LC3-I is transformed into LC3-II, which requires the participation of ATG7, ATG12, ATG5, and ATG16L1. The ER membrane is derived to form double-vesicular organelles termed autophagosomes to isolate parts of the cytoplasm and wrap the cargos. Then, p62 transports the ubiquitinated proteins to the autophagosomes before the fusion of autophagosome with lysosome to form the autolysosome. During the fusion, the inner vesicle and enclosed cargo are degraded by hydrolases that ordinarily resident in the lytic compartments $^{38,39}$ (Figure 1).

Although the selective autophagy process is largely consistent with non-selective, unlike the latter, the autophagosomes in selective autophagy are closely around the substrates, which enables the degradation of certain molecules or organelles. Besides that, some factors provide key links in ensuring its selectivity. Firstly, ULK1 kinase complexes cluster around certain cargoes for selective autophagy rather than organize supramolecular network. Secondly, autophagy receptors are bound to designated cargos or conditionally recruited to their cargos. This process depends on a specific surface signal, for example, ubiquitination of surface proteins of targeted organelles via 


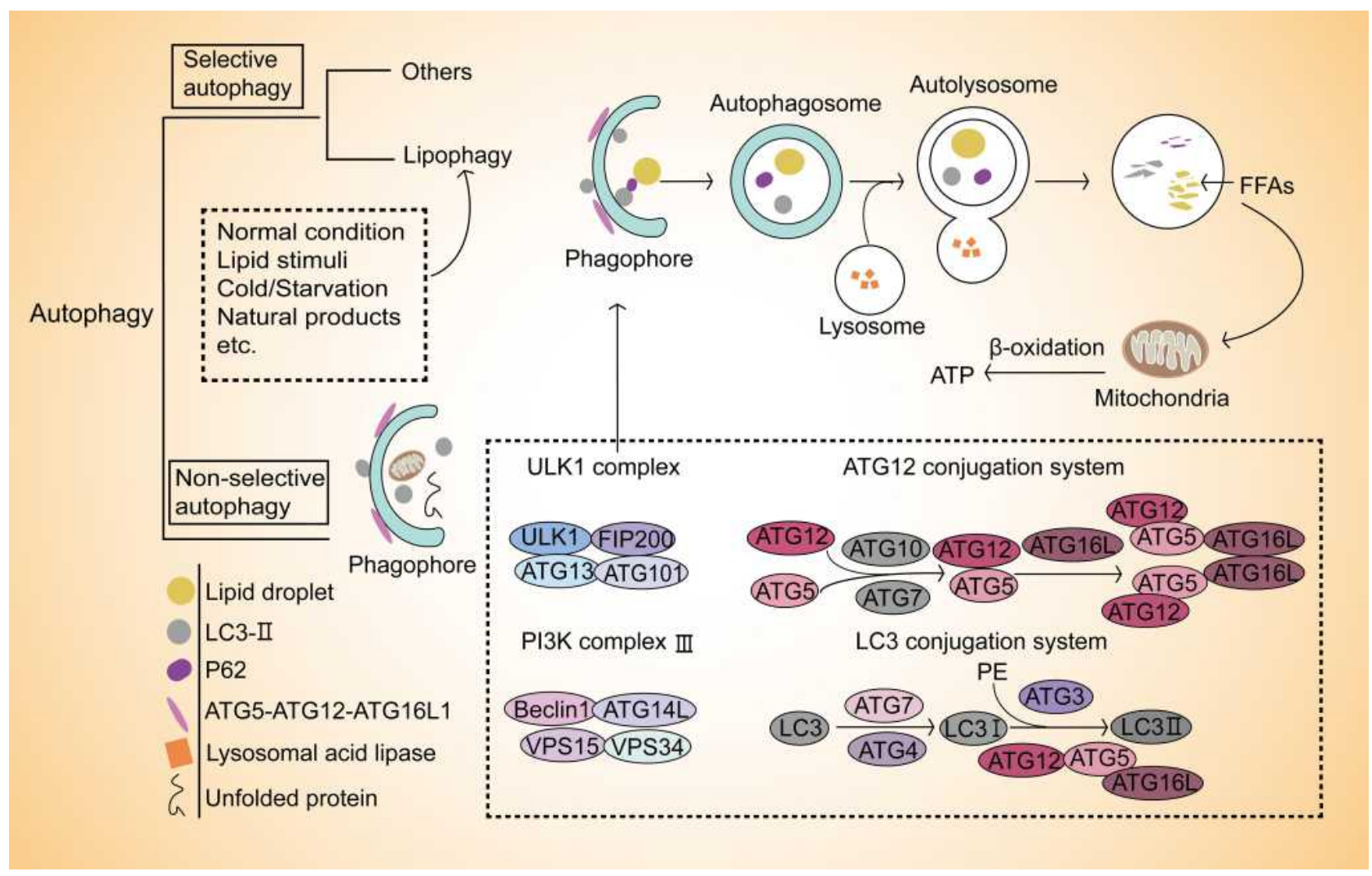

Figure I Lipophagy selectively breaks down lipid droplets. Autophagy widely removes damaged organelles or unfolded proteins non-selectively. Compared with nonselective, autophagy also occurs in a selective manner. Lipophagy is one of the selective autophagy that occurs in several conditions including normal condition, lipid/cold/ starvation stimuli or natural products induction, etc. It selectively breaks down lipid droplets then fuels $\beta$-oxidation with free fatty acids (FFAs) in mitochondria through autophagy-lysosome pathway. The formation of phagophore is the initiation and key of this process that involved in ULKI complex, ATGI2 conjugation system, PI3K complex III and LC3 conjugation system.

binding to small ubiquitin-like modifiers to mediate the formation of selective autophagosomes. In a recent report, only adipose triglyceride lipase (ATGL) and patatin-like phospholipase domain containing 8 have been identified as lipophagy receptors in mammals. ${ }^{12}$ Thirdly, ATG8 family proteins generate a high-affinity binding surface for proteins to ensure selectivity. ${ }^{38}$

\section{Lipid Droplet is a Major Vehicle Designed for Lipid Homeostasis and a Target for T2DM Treatment}

LD is the target of lipophagy and a major vehicle for lipid homeostasis. It is the main lipid storage site for cells and plays an essential role in lipid metabolism. With sufficient intracellular nutrition the lipids are generally converted into neutral lipids to store in LDs in adipocytes, such as TGs and cholesterol esters. ${ }^{32}$ Otherwise, the lipids stored in LDs are hydrolyzed into FFAs for the purpose of providing fuel for $\beta$-oxidation in mitochondria when cells are deficient in nutrition. Intracellular accumulation of LDs is a hallmark of several metabolic syndromes. ${ }^{40}$ Situation varies according to the excessive lipids. Cells subsequently synthesize a large number of LDs in other tissues, which is called ectopic lipid. It leads to disorders of lipid metabolism and lipid toxicity, including abnormal lipoprotein metabolism, insulin dysfunction and altered insulin secretion $^{41}$ (Figure 2).

The most relevant tissues in this process are liver, skeletal muscle and islets, respectively. Liver is the major site of lipogenesis and lipid oxidation, and it is a central organ in lipid metabolism. ${ }^{13}$ The abnormal accumulation of lipids in LDs of the liver is a characteristic of various liver diseases that are always related to insulin resistance. $^{23}$ The elevated lipid accumulation in liver leads to a higher lipid export, which then increases fat accumulation in skeletal muscle and the intra-pancreatic fat. Skeletal muscle is responsible for the body's energy consumption, participating in thermogenesis, glucose and lipid uptake, and other metabolic processes. ${ }^{42}$ 


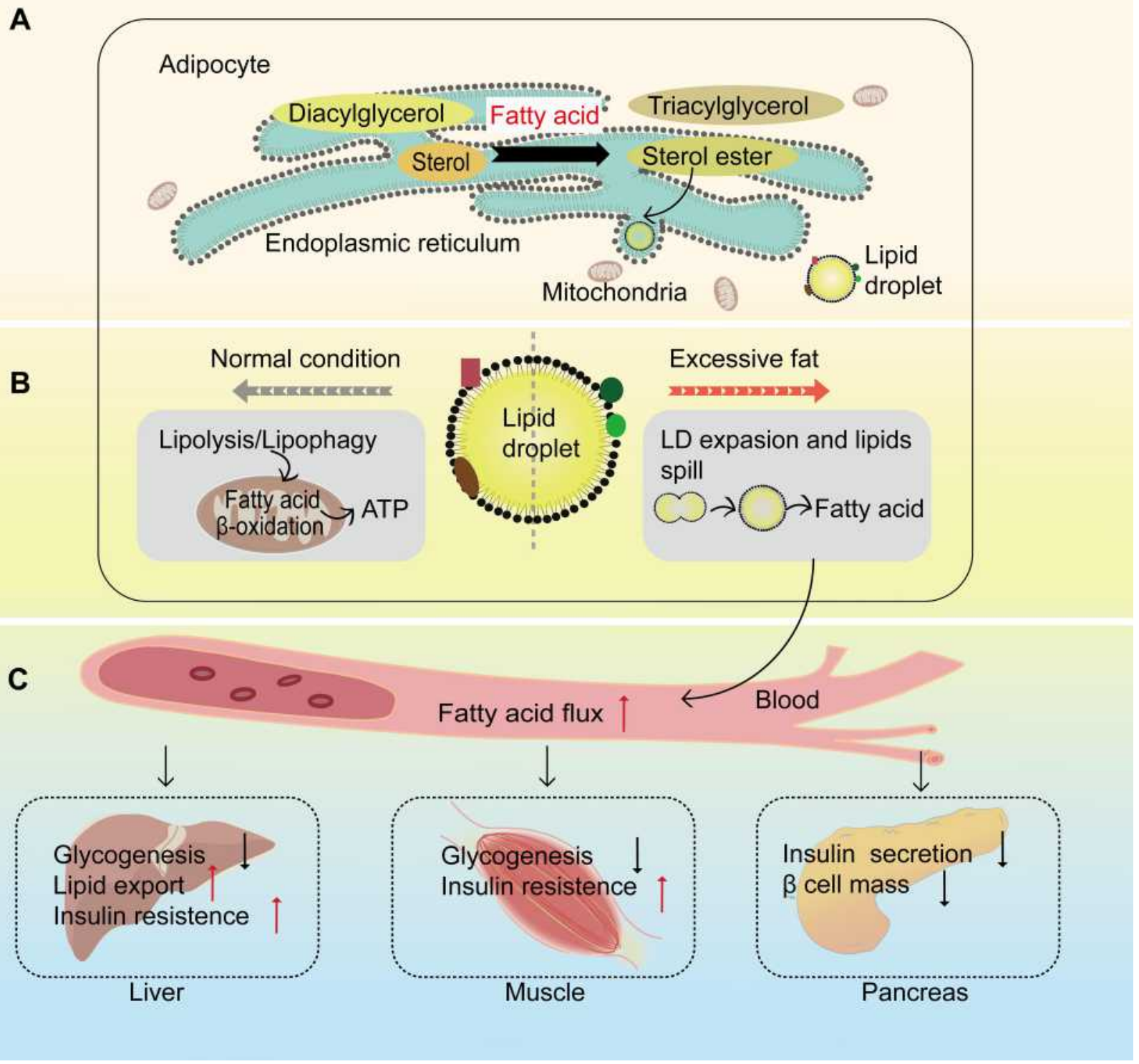

Figure 2 Lipid droplet is a major vehicle designed for lipid homeostasis. (A) In the adipocytes, excess fatty acids are converted into neutral lipids for storage in lipid droplets (LDs). Lipids derived from the de novo glycerolipid synthesis or recycling of diacylglycerols and sterols that generated by LDs degradation synthesize nascent LDs in the endoplasmic reticulum. (B) These LDs have different outcomes under distinct conditions. Normally, these LDs provide fuel for $\beta$-oxidation in mitochondria to release ATP through lipolysis or lipophagy. With excessive fat, these LDs expand through LD-LD fusion till they exceed their capacities to accommodate lipids. (C) The excessive lipids spill into the blood, followed by increasing fatty acid flux, then export to different tissues like liver, muscle and pancreas, which is a major contributor to reduced glycogenesis and increased insulin resistance in liver and muscle as well as increased liver lipid export, reduced insulin secretion and the loss of pancreatic $\beta$ cells.

Intramyocellular TGs are stored in LDs as an important site of energy storage and a fuel source in skeletal muscle. An imbalance between intramyocellular TGs degradation and fatty acids oxidation might lead to lipotoxicity and insulin resistance. ${ }^{5}$ The degree of insulin resistance in T2DM patients is positively correlated with the size of LD in skeletal muscle. ${ }^{43}$ Additionally, ectopic fat accumulation in the pancreas plays an important role in the onset of T2DM. ${ }^{25} \beta$-cell dysfunction is related to increased intra- pancreatic fat. Meanwhile, some changes are also observed in T2DM patients with abnormal accumulation of lipids in LDs in the $\beta$-cells including significant changes in the gene expression of lipid metabolism, apoptosis and oxidative stress. ${ }^{19}$ Besides the above, LDs are also stored in the intestine, and its existence is not only a lipid pool but also a potential pathogenic factor, because its existence affects gut microbiota, ${ }^{44}$ which has been proved to be closely related to the pathogenesis of T2DM. 
Abnormal lipid metabolism is closely related to abnormal LDs. ${ }^{45}$ These LDs contribute to T2DM when they are stored in peripheral tissues or $\beta$-cells. Therefore, LD as a major vehicle designed for lipid homeostasis could be a target for T2DM treatment.

Lipophagy could connect T2DM with lipid dysregulation through LDs. It could play the same role as nutrient restriction which has been demonstrated to be the most efficient strategy in reducing visceral fat in both animals and humans. ${ }^{46,47}$ Furthermore, a clinical trial conducted by The Diabetes Remission Clinical Trial also confirmed the benefits of nutrient restriction by weight loss after several steps. These treatments ultimately lead to a major fall in liver fat export and intra-pancreatic fat which are link to the remission of T2DM. ${ }^{48}$ Therefore, lipophagy might be a potential therapeutic target of T2DM.

\section{Effects of Natural Products on T2DM-Related Tissues Through Lipophagy}

As previously mentioned, lipophagy breaks down LDs through the autophagy-lysosome pathway for either energy requirements or removing excessive fat from tissues to restore the normal function. To downregulate fat deposition, the ability to remove LDs is more concerned rather than its ability to supply energy. Therefore, the latter makes a greater effort to reduce the ectopic fat in liver, skeletal muscle and $\beta$-cells when natural products modulate lipophagy to improve the T2DM-related lipid excess.

To investigate the effects of natural products in removing LDs through autophagy-lysosome pathway and what changes have taken place in tissues or cells, rational models and experiment projects are prerequisites for further detection. In previous studies, use of HFD to animals or oleic acid and/or palmitic acid to cells effectively promote the accumulation of lipid. Some obesity mouse models, such as $\mathrm{db} / \mathrm{db}$ mice, ${ }^{49}$ are also taken into consideration. Additionally, homozygous green fluorescent protein-LC3 transgenic mice are selected to detect autophagy flux after HFD, which reflects the true level of autophagy. ${ }^{50}$ In the actual study, there are two basic problems: the changes of fat accumulation of insulin target tissues and $\beta$-cells and the expression of lipophagy-related genes and proteins. So based on these two problems, some experiments are carried out. It is concluded that lipophagy decreases lipid accumulation after the Oil red $\mathrm{O}$ staining and biochemical detection including triglyceride of cells or tissues and serum total cholesterol, high- and low-density lipoprotein cholesterol and alanine aminotransferase. Meanwhile, in order to confirm that these natural products reduce fat accumulation through lipophagy, the autophagy-related proteins and mRNA expression and autophagy flux are important indicators to assess the turnover of lipids stored in LDs. For example, it is well known that AMPK is a nutrient sensor and acts on cellular lipid metabolism. Meanwhile, AMPK shows a crucial role in lipophagy induction, which means that its expression should be measured. More importantly, the co-localization of LDs and autophagosomes is more direct evidence for this pathway.

Natural products regulated lipophagy to reduce fat accumulation through various mechanisms (Table 1). Resveratrol is a natural polyphenol that widely exists in grapes and peanuts. And this compound is also found in some medicinal plants (Polygonum cuspidatum Sieb.et Zucc. and Cassia tora Linn.). Male Wistar rats were fed HFD before resveratrol $(200 \mathrm{mg} / \mathrm{kg} . \mathrm{bw})$. At the end of administration of 18 weeks, the lipid contents in liver were measured by Oil Red O staining and the bioassay of metabolic parameters. Hepatic mRNA expressions in rats were also measured to identify the effects of resveratrol. These results showed that resveratrol could significantly increase autophagy and sirtuin 1 (SIRT1) activity, decrease lipid accumulation and stimulate fatty acid $\beta$ oxidation in hepatocytes by inducing lipophagy via the cyclic adenosine monophosphate (cAMP)-protein kinase A (PRKA)-AMPK-SIRT1 signaling pathway. ${ }^{51}$

Quercetin is a flavonoid with multiple phenolic hydroxyl groups and exists in many plants including Sophora japonica L., and Platycladus orientalis (L.) Franco and other medicinal plants. Quercetin induces lipophagy to reduce lipid accumulation both in vivo and in vitro. Male Sprague Dawley rats were fed with HFD for model establishment, then co-administration with quercetin $(100 \mathrm{mg} / \mathrm{kg} . \mathrm{bw})$ for 8 weeks. Serum and hepatic samples were collected finally for determination of lipid parameters including total cholesterol, high- and lowdensity lipoprotein cholesterol, and very low-density lipoproteins. Except for lipid parameters, tissue samples were collected for related proteins detection. In vitro, hepatocytes were treated with $1 \mathrm{mM}$ FFA (the basic compositions were palmitic acid and oleic acid in the proportion of 1:2), which followed by quercetin for $24 \mathrm{~h}$ before cells were collected for bioassays as the tissue samples. It was demonstrated that the inositol-requiring enzyme-1 $\alpha /$ X-box binding protein 1 pathway-associated lipophagy 
Table I Models and Signaling Pathways Involved in Typical Natural Products in Lipophagy Induction

\begin{tabular}{|c|c|c|c|}
\hline Category & $\begin{array}{l}\text { Natural } \\
\text { Product }\end{array}$ & Experiment Model & $\begin{array}{l}\text { Signaling } \\
\text { Pathway }\end{array}$ \\
\hline \multirow[t]{3}{*}{ Terpenoids } & Ginsenoside Rb2 & HepG2 cell; Mouse primary hepatocyte; Male mice (C57BL/KsJ-Lepdb) ${ }^{49}$ & AMPK/SIRTI \\
\hline & Ursolic acid & HepG2 cell ${ }^{65}$ & AMPK \\
\hline & $\begin{array}{l}\text { Iridoids of } \\
\text { Valeriana fauriei }\end{array}$ & Huh7 cell; Atg7-/- immortalized MEF cell ${ }^{67}$ & NA \\
\hline \multirow[t]{2}{*}{ Alkaloids } & Berberine & $\begin{array}{l}\text { HepG2 cell; Mouse primary hepatocyte; Male mice (liver-specific SIRTI knockout) fed with } \\
\text { high fat high sucrose diet }{ }^{54}\end{array}$ & SIRTI \\
\hline & Caffeine & HepG2 cell; Male mice (C57BL/6) fed with high fat diet ${ }^{56}$ & mTOR \\
\hline \multirow[t]{4}{*}{ Flavonoids } & Kaempferol & RIN-5F cell; Rat primary islets cell ${ }^{17}$ & AMPK/mTOR \\
\hline & Formononetin & HepG2 cell $^{70}$ & TFEB \\
\hline & $\begin{array}{l}\text { Extracts of } \\
\text { bilberry fruits }\end{array}$ & Alpha mouse liver 12 cell $^{72}$ & Rubicon/p62 \\
\hline & Quercetin & HepG2 cell; Male rat (Sprague Dawley) fed with high fat $\operatorname{diet}^{52,53}$ & IREI $\alpha / \mathrm{XBPIs}$ \\
\hline \multirow[t]{3}{*}{ Polyphenols } & Resveratrol & HepG2 cell; Mice (I29/SvJ) fed with high fat $\operatorname{diet}^{51}$ & $\begin{array}{l}\text { CAMP-PRKA- } \\
\text { AMPK-SIRTI }\end{array}$ \\
\hline & $\begin{array}{l}\text { Epigallocatechin- } \\
\text { 3-Gallate }\end{array}$ & HepG2 cell; Huh7 cells; Male mice (C57BL/6) fed with high fat diet ${ }^{75}$ & AMPK \\
\hline & $\begin{array}{l}\text { Bergamot poly- } \\
\text { phenol fraction }\end{array}$ & Male rat (Han WIST) fed with cafeteria $\operatorname{diet}^{76}$ & NA \\
\hline Steroid & Dioscin & Male mice (C57BL/Ksj-Lepdb) fed with high fat diet ${ }^{77}$ & $\begin{array}{l}\text { p-mTOR/ } \\
\text { mTOR }\end{array}$ \\
\hline \multirow[t]{2}{*}{ Others } & Trehalose & $\begin{array}{l}\text { HepG2 cell; Mouse primary hepatocyte (ATGI6LI }{ }^{\mathrm{HM}} \text { knock-in mice); Mouse primary } \\
\text { hepatocyte (MTTP-knockout); Male and female mice (C57BL/6)) fed with high-fructose diet }{ }^{79}\end{array}$ & AMPK/ULKI \\
\hline & Vitamin D3 & Male mice (C57BL/6]) fed with high fat diet ${ }^{60}$ & $\begin{array}{l}\text { AMPK/SIRTI/ } \\
\text { PGC-I }\end{array}$ \\
\hline
\end{tabular}

Abbreviations: NA, not available; AMPK, adenosine 5'-monophosphate (AMP)-activated protein kinase; SIRTI, sirtuin I; mTOR, mammalian target of rapamycin; TFEB, transcription factor EB; IREI $\alpha$, inositol-requiring enzyme-I $\alpha$; XBPIs, X-box binding protein I; PRKA, protein kinase A; p-mTOR, phosphorylated-mammalian target of rapamycin; ULKI, UNC5I-like kinase complex I; PGC-I, peroxisome proliferators activated receptor-gamma I.

in quercetin-mediated protection against FFA-stimulated hepatic lipid deposition. ${ }^{52}$ Beyond that, quercetin promotes lipophagy by decreasing perilipin 2 (Plin2) level, as well as the activated AMPK activity and increased colocalization of liver LC3-II and Plin2 proteins. $^{53}$

Berberine, which is mostly isolated from Coptis chinensis Franch. and Phellodendron chinense Schneid, is an alkaloid with lipid- and glucose-lowering properties. ${ }^{54}$ Known as a useful agent for pancreatic $\beta$-cells, such as the regulation of insulin secretion, berberine also lowers lipid though inducing lipophagy and represses lipid accumulation in hepatocytes. Male C57BL/6 mice were fed with high-fat, high-sucrose diet and then treated with berberine $(5 \mathrm{mg} / \mathrm{kg} /$ day $)$. The administration of berberine caused the reduction of Oil Red O staining area, which implied the reduction of LDs. Meanwhile, it induced the elevation of autophagy through the observation of membrane-associated and PE-conjugated form of LC3-II. ${ }^{18}$

Caffeine is a main functional component in green tea. It has been demonstrated to inhibit fat accumulation in 3T3-L1 cells. ${ }^{55}$ Except that, it has an effect on the prevention and treatment of liver diseases by activation of lipophagy to lower levels of hepatic lipids. The mobilization and hydrolysis of TGs to FFAs lead to increased delivery of FFAs to the mitochondria for $\beta$-oxidation through the autophagy-lysosomal pathway. ${ }^{56}$ 
Ginsenoside Rb2, a key component of Panax ginseng, has the efficiency to modulate energy input, lipid synthesis and energy consumption in skeletal muscle and liver via the AMPK pathway. In vitro, Rb2 $(50 \mu \mathrm{mol} / \mathrm{L})$ was used in HepG2 cells and primary mouse hepatocytes to increase autophagic flux. It alleviated hepatic lipid accumulation by restoring autophagy to reduce lipid content in liver through increasing expression of SIRT1 and phosphorylation of AMPK in cultured steatotic hepatocytes and resulted in improved NAFLD and glucose tolerance. ${ }^{57}$

Given that excessive fat in liver is generally involved in insulin resistance, and it can redirect glucose from glycogen synthesis to lipogenesis. ${ }^{58}$ Regulating lipophagy in liver to remove the excessive fat can improve liver diseases, insulin resistance and glycogen synthesis, ${ }^{59}$ which alleviates T2DM.

In addition, some other natural products are applied to promote lipophagy in $\beta$-cell. Kaempferol is a natural flavonoid. Recently, it has been shown that kaempferol prevents ectopic lipid accumulation and ER stress through AMPK-mediated lipophagy to restore $\beta$-cell function. ${ }^{17}$

Another agent to regulate lipophagy is vitamin D3 which is involved in lipid metabolism, insulin secretion, and inflammation. It is found that vitamin D3 supplementation not only improves glucose intolerance but also plays a favorable role in the improvement of lipolytic mechanisms, including $\beta$-oxidation, lipophagy, and inhibition of lipid synthesis in T2DM. ${ }^{52}$

It is suggested that metformin, a natural product derivative that is the first-line drug for T2DM, can induce autophagy and mimic the state of nutrient restriction. As an oral biguanide, metformin is widely used in obese people or T2DM patients to ameliorate hyperglycemia by improving peripheral sensitivity to insulin and reducing gastrointestinal glucose absorption and hepatic glucose production. ${ }^{61}$ Surprisingly, when investigating the role of forkhead homeobox type protein $\mathrm{O} 1$ (FoxO1) in modulating lysosomal lipid catabolism during nutrient restriction in adipocytes, metformin is certified as a pro-lipolytic drug via the induction of FoxO1-mediated lipophagy in adipose tissue. $^{62}$ Meanwhile, metformin activates lipophagy to clear LDs through activation of AMPK and phosphorylation of ULK1 and Beclin1. ${ }^{63}$

As mentioned above, natural products inducing lipophagy include flavonoids, polyphenols, terpenoids and alkaloids, etc (Table 2). Among them, flavonoids and polyphenols are the most common compounds used in lipophagy activation. In previous studies, different flavonoids have been demonstrated to activate AMPK in vitro and in vivo to trigger lipophagy. ${ }^{80}$ Similar results have been observed in many polyphenols. ${ }^{43}$ Compared with lipophagy, different mechanisms are also involved in the lipid metabolism by flavonoids and polyphenols. For example, the bergamot polyphenol fraction contains two rare flavonoids acting as direct 3-hydroxy-3-methylglutaryl coenzyme A reductase inhibitors, with function of downregulating cholesterol synthesis. ${ }^{80}$ Flavonoids with the hydroxylated phenolic structure sometimes act as a kind of anti-diabetic drugs due to anti-inflammatory, attenuating insulin resistance, increasing glycogen synthesis, and ameliorating islets dysfunction effects. ${ }^{81}$ The structures of these polyphenols are characterized by a different number of phenolic hydroxyl groups, which could neutralize the acid or remove the free radicals. ${ }^{73}$ However, the relationships of these structures and their functions of inducing lipophagy have not been elucidated.

Some terpenoids and alkaloids are relatively common in the application of activating lipophagy. Alkaloids have attracted abundant attention in regulating lipid metabolism. ${ }^{82}$ They are alkaline nitrogenous organic compounds, which have complex cyclic structures and exhibit significant activities in lipid metabolism. ${ }^{82}$ Terpenoids interact with different metabolic pathways, exerting beneficial effects against many metabolic diseases through the modulation of lipid transport and adipose tissue deposition. ${ }^{83}$ Some terpenoids lower excessive LDs through lipophagy possibly due to their lipophilicity. Additionally, some of these compounds trigger additional mechanisms such as the increase in insulin sensitivity, anti-oxidation and anti-inflammation, which makes them ideal candidates to improve T2DM.

Therefore, lipophagy could be considered when screening the active components or studying the mechanisms of natural products with the glucose- and lipid-lowering effects. With the help of pharmaphylogeny, it is more convenient to find candidate medicinal plants. The activities of these nonlipophagy natural products might be structure dependent. ${ }^{81}$ However, it is not clear whether their regulations of lipophagy are related to structures. More researches about the relationships between structures and lipophagy are needed to clarify characteristics of these lipophagy-dependent natural products in the future. Meanwhile, there is a lack of knowledge about natural products in the treatment of T2DM through lipophagy, such as changes in blood glucose during this process. Therefore, more animal studies, human studies, or cellular studies are needed for more details in this area. 
Table 2 Structures and Primary Source of Typical Natural Products in Lipophagy Induction

\begin{tabular}{|c|c|c|c|}
\hline Category & Natural Product & Structure & Primary Source \\
\hline \multirow[t]{3}{*}{ Terpenoids } & Ginsenoside Rb2 & & Panax ginseng C. A. Meyer ${ }^{64}$ \\
\hline & Ursolic acid & & Ligustrum lucidum Ait.; Eriobotrya japonica(Thunb.)Lindl. ${ }^{66}$ \\
\hline & Iridoids of Valeriana fauriei & NA & Valeriana fauriei ${ }^{67}$ \\
\hline \multirow[t]{2}{*}{ Alkaloids } & Berberine & & Coptis chinensis; Phellodendron amurense; Hydrastis canadensis ${ }^{68}$ \\
\hline & Caffeine & & Coffea Linn; Camellia sinensis (L.) O. Ktze. ${ }^{56}$ \\
\hline \multirow[t]{4}{*}{ Flavonoids } & Kaempferol & & Kaempferia galanga Linn. ${ }^{69}$ \\
\hline & Formononetin & & Trifolium pratense; Astragalus membranaceus ${ }^{71}$ \\
\hline & Extracts of bilberry fruits & NA & Vaccinium myrtillus $\mathrm{L}^{72}$ \\
\hline & Quercetin & & Capparis spinosa L.; Piper Nigrum L. ${ }^{73}$ \\
\hline \multirow[t]{3}{*}{ Polyphenols } & Resveratrol & & Veratrum grandiflorum ${ }^{74}$ \\
\hline & Epigallocatechin-3-gallate & & Camellia sinensis (L.) O. Ktze. ${ }^{75}$ \\
\hline & Bergamot polyphenol fraction & NA & Citrus medica L. var. sarcodactylis Swingle ${ }^{76}$ \\
\hline
\end{tabular}

(Continued) 
Table 2 (Continued).

\begin{tabular}{|c|c|c|c|}
\hline Category & Natural Product & Structure & Primary Source \\
\hline Steroid & Dioscin & & Dioscorea spongiosa ${ }^{78}$ \\
\hline \multirow[t]{2}{*}{ Others } & Trehalose & & Bacteria; yeast; fungi \\
\hline & Vitamin D3 & & Animal livers \\
\hline
\end{tabular}

Abbreviation: NA, not available.

\section{Discussion}

Here we describe that natural products induce lipophagy providing us with an insight into T2DM treatment (Figure 3). Activated lipophagy reduces the LDs in peripheral tissues and $\beta$-cells to improve insulin resistance or restore $\beta$-cells function, which is beneficial for insulin secretion and sensitivity.

The accumulation of LDs has become the crucial object in lipid metabolism and already attracted plenty of interest in diseases. ${ }^{83}$ For example, the development of fatty liver diseases, obesity, atherosclerosis, T2DM. ${ }^{40,85}$ The changes related to lipolysis and fat mobilization are the main causes of these dyslipidemia-related diseases. Therefore, as an important role in the process, the LDs need to sustain the number and size within a reasonable level. Some factors are involved in the process. Firstly, changes in the phospholipid ratio of the LD membrane compositions mainly affect the synthesis, maturation, and degradation of LD. ${ }^{31}$ Secondly, some coated proteins or organelles are related to the formation and digestion of LDs. For example, acyl-CoA synthetase long-chain family member 3 physically interacts with other LD proteins that are conversely crucial adaptor proteins, which determines the size and numbers. Additionally, PAT family proteins play distinct roles in stabilizing LD nucleation and lipid storage. ${ }^{84,86}$ Take PLIN1 and PLIN2 as examples, they are well known for reducing the association of ATGL with LDs to slow TG turnover and protect LDs from lipolysis. The organelles that connect to LDs take part in the transformation of LDs, such as the ER. The factors provide here only cover some important ones, which have been discussed in more detail. ${ }^{23}$ These factors mentioned above might be the key points to solve the stability of LDs, which is important for these diseases caused by abnormal lipid metabolism. ${ }^{87}$

However, in this review, we are more concerned about the process that lipophagy breaks down the LDs with ectopic deposition in peripheral tissues and $\beta$-cells. It has been proven that lipophagy has been adopted in liverrelated diseases, neurodegenerative diseases or lipid storage myopathy ${ }^{88}$ before the pivotal role of lipophagy in lipid metabolism emerges. ${ }^{32}$

Current evidence suggests that lipophagy cooperates with classic lipolysis to regulate lipid metabolism. ${ }^{89}$ There are some similarities between lipophagy and lipolysis when they play the role in degrading LDs. Firstly, no matter what kind of pathway for LDs degradation, there are both regulated by the protein composition on the 


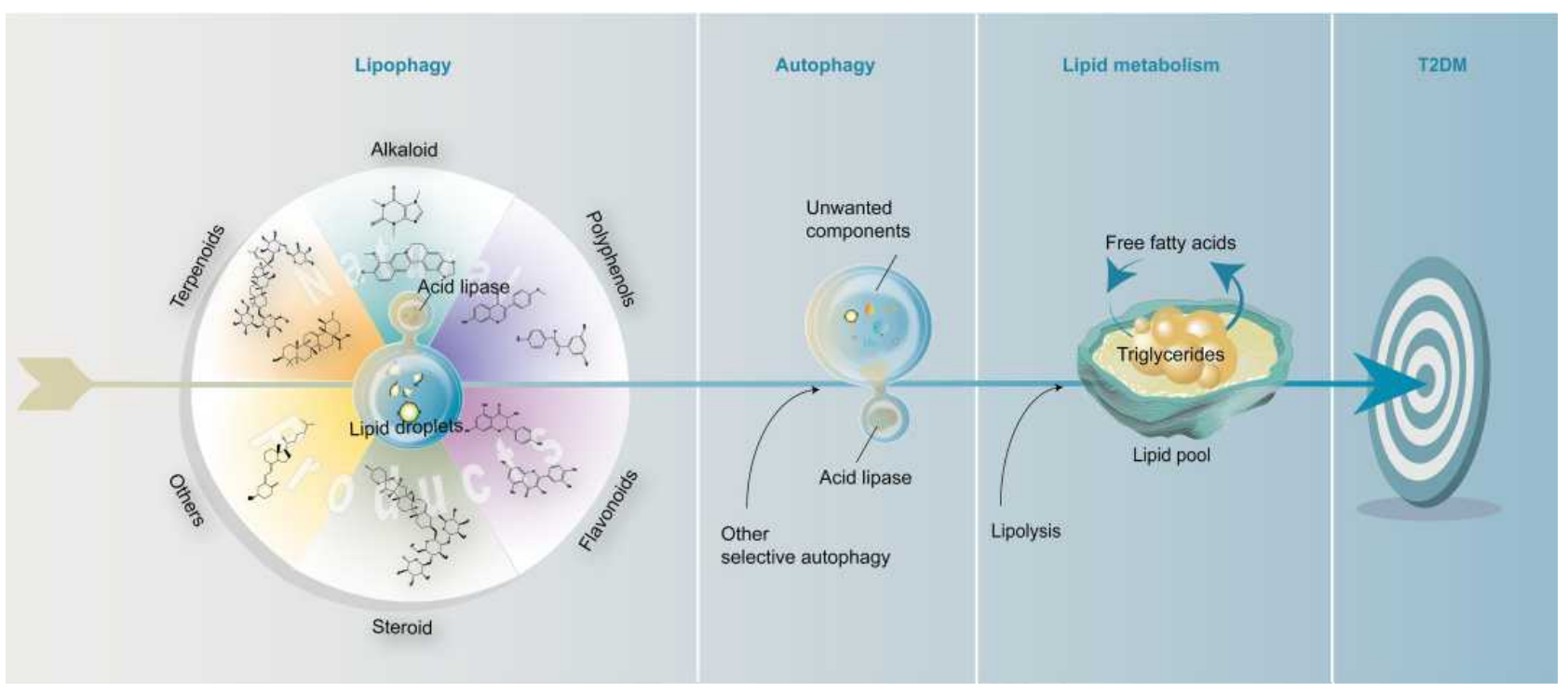

Figure 3 Lipophagy provides a new perspective of natural products in T2DM treatment. Lipid droplets are considered as the targets of lipophagy. During this process, various natural products are targeted to the initiation of lipophagy. The activated lipophagy subsequently cooperates with other selective autophagy to exert the positive role of autophagy in lipid metabolism by removing unwanted components. Together with lipolysis, it finally achieves the goal of treating T2DM by regulating lipid metabolism. It is anticipated that a novel therapeutic strategy for T2DM could be considered following the route of "natural product-lipophagy-autophagy-lipid metabolism-T2DM".

surface of LDs. Secondly, both pathways occur in multiple tissues and the products are same in supplying substrates for $\beta$-oxidation and cellular biosynthesis. ${ }^{90}$

However, the differences are as follows. The enzymes that catalyze this process are different. These two pathways hydrolyze TGs in the presence of neutral lipase and acid lipase, respectively. Meanwhile, lipolysis is implemented step by step with different enzymes, such as ATGL, hormone-sensitive lipase and monoglyceride lipase. ${ }^{90}$ Lipophagy forms autophagosomes to wrap LDs and deliver them to lysosome for degradation. Additionally, cross-talk of lipolysis and lipophagy exists. Lipophagy is essential in LDs catabolism during lipolysis for recruitment on LDs and the initiation of lipolysis because ATGL cannot facilitate this process without lipophagy and possesses LC3-II interaction motifs. ${ }^{58}$

Furthermore, the metabolism of lipids by lipophagy suggests a new mechanism by which autophagy may act to prevent cell death, ${ }^{31}$ which is benefit to $\beta$-cell survival. In previous research, the small GTPases have been demonstrated to regulate lipophagy. It is represented by Rab7 and Rab10, which participate in cellular traffic events during lipophagy. Beyond, Rab7 has been found to be required in autolysosome formation. ${ }^{91,92}$ And Rab25 combines with PI3KCIII, which directs the process of autophagy to recognize, wrap and degrade LDs. ${ }^{93}$ The transcriptional regulation of this pathway has received with concern. Transcription factor EB as well as transcription factor E3 regulates lysosomal and autophagy genes, which activates lipophagy. ${ }^{94}$ All of these are the foundations of lipophagy in lipid metabolism.

To date, the gains have been made over the past decade in the dysregulation of autophagy in onset of T2DM. ${ }^{38}$ The application of other selective autophagy in T2DM is rarely studied yet. Autophagy is crucial for $\beta$-cell owing to its function to remove misfolded proteins during synthesis of proinsulin in ER which inhibits $\beta$-cell stress and apoptosis. ${ }^{3}$ Defective autophagy contributes to impaired $\beta$ cell function and development of insulin resistance. ${ }^{95-97} \mathrm{In}$ this period, at least in part, organelles like mitochondria or ER play a crucial role in $\beta$-cell survival/death, insulin secretion and insulin action/sensitivity that rely on autophagy. ${ }^{98}$ ER stress has been identified as a contributor to insulin resistance resulting from its direct inhibitory effect on insulin signaling and indirect effect on lipid accumulation. ${ }^{99}$ Impaired autophagy leads to ER stress, followed by decreasing the number and/or proliferation of $\beta$-cell, which ultimately causes hyperglycemia, glucose intolerance and hypoinsulinemia. ${ }^{100}$ Therefore, it is also important to consider the application of other selective autophagy for the improvement of T2DM when these organelles are damaged.

Lipid deposition in $\beta$-cells is one of the major causes of loss of $\beta$-cell mass and function. ${ }^{12}$ To reduce fat deposition in $\beta$-cells is a strategy for us to restore the function of $\beta$-cells. Lipophagy is a way to reduce lipid 
deposition in $\beta$ cells. Yet it's worth noting that some studies reach the opposite conclusion. On the one hand, a study about the kaempferol ameliorating palmitic acidinduced pancreatic $\beta$-cell injury, kaempferol decreased the $\beta$-cell fat deposition while simultaneously promoted glucose stimulated insulin secretion. ${ }^{17}$ Lipophagy improves insulin secretion by regulating lipid metabolism in $\beta$-cells. On the other hand, lipolysis increased insulin secretion when autophagy and lysosomal acidic lipase in MIN6 cells and primary islets was chronic ( $\geq 8 \mathrm{~h}$ ) inhibited in the way of pharmacology and/or gene. This phenomenon was more obvious after high glucose stimulation, which possibly means that lipophagy is unfavorable for glucose-stimulated insulin secretion. It was possibly because that lipophagy depleted substrate for the neutral lipases, which were activated acutely by glucose. ${ }^{8}$ Therefore, more studies will be required before we know more about the cross-talk between lipophagy and lipolysis.

Besides, some issues also remain to be addressed in the further study of lipophagy. Firstly, it is known that the disturbed autophagy cannot remove the damaged organelles or aggregated proteins caused by lipotoxicity in time, leading to the pathogenesis of T2DM, which indicates that the restoration of disturbed autophagy is crucial for the T2DM treatment. ${ }^{101}$ Given that some common key proteins are participated in both non-selective autophagy and lipophagy, like LC3 and p62, whether the nonselective autophagy can be simultaneously restored when enhancing lipophagy is an important issue. Otherwise, it should focus more on the induction of other selective autophagy such as glycophagy which enables cells to salvage key metabolites to sustain and facilitate core anabolic functions. ${ }^{36}$ Moreover, lipophagy could be triggered during starvation by exercise and energy-restrictive diet, between which their relationship remains to be further clarified. ${ }^{102}$ More importantly, it should be concerned that the FFAs released from LDs by lipophagy might be accumulated if not being consumed in mitochondria, which leads to lipotoxicity. Therefore, in previous studies, $\beta$-oxidation was deliberately up-regulated at the same time. To ensure the efficiency of consuming the excessive FFAs, even the mitophagy that responds to damaged mitochondria could be concerned. Lastly, it has been demonstrated that visceral fat is much more important in the onset of T2DM, than subcutaneous fat. More studies are needed to determine the target preference of lipophagy in ectopic lipid clearance. ${ }^{103}$

\section{Conclusions}

Lipophagy is suggested to improve T2DM by lowering lipid contents of insulin-targeted tissues and $\beta$-cells. Meanwhile, it is a new target for natural products in T2DM treatment. These natural products include various compounds which trigger lipophagy through AMPK, SIRT1, mTOR, etc. It is accepted that traditional Chinese medicine has advantages in treating T2DM, with the mechanisms of a large number of natural products not being elucidated. Lipophagy provides a new insight for screening bioactive agents to treat T2DM and clarifying their underlying mechanisms, which would be a breakthrough for future research on natural products to improve diabetes. Despite lipophagy success in decreasing lipid accumulation in vivo and in vitro, further studies are still needed to be carried to verify practical application in humans.

\section{Acknowledgments}

This work was supported by the National Natural Science Foundation of China (grant number 81773891, 82004011), the National Great New Drugs Development Project of China (grant number 2017ZX09301-040), the National Key Research and Development Program of China (grant number 2020YFF01014606), the Beijing Talents Youth Top Team Project (grant number 2018000021223TD09), and the 'Beijing Hospitals Authority' Ascent Plan (grant number DFL20190702) .

\section{Disclosure}

The authors report no conflicts of interest in this work.

\section{References}

1. Salunkhe VA, Veluthakal R, Kahn SE, et al. Novel approaches to restore beta cell function in prediabetes and type 2 diabetes. Diabetologia. 2018;61(9):1895-1901. doi:10.1007/s00125-018-4658-3

2. Sabag A, Way KL, Keating SE, et al. Exercise and ectopic fat in type 2 diabetes: a systematic review and meta-analysis. Diabetes Metab. 2017;43:195-210. doi:10.1016/j.diabet.2016.12.006

3. Barlow AD, Thomas DC. Autophagy in diabetes: $\beta$-cell dysfunction, insulin resistance, and complications. DNA Cell Biol. 2015;34 (4):252-260. doi:10.1089/dna.2014.2755

4. Shulman GI. Ectopic fat in insulin resistance, dyslipidemia, and cardiometabolic disease. $N$ Engl $J$ Med. 2014;371(12):1131-1141. doi:10.1056/NEJMra1011035

5. Badin PM, Langin D, Moro C. Dynamics of skeletal muscle lipid pools. Trends Endocrinol Metab. 2013;24:607-615. doi:10.1016/j. tem.2013.08.001 
6. Condello M, Pellegrini E, Caraglia M, et al. Targeting autophagy to overcome human diseases. Int J Mol Sci. 2019;20(3):1-27. doi:10.3390/ijms20030725

7. Madrigal-Matute J, Cuervo AM. Regulation of liver metabolism by autophagy. Gastroenterology. 2016;150:328-339. doi:10.1053/ j.gastro.2015.09.042

8. Pearson GL, Mellett N, Chu KY, et al. Lysosomal acid lipase and lipophagy are constitutive negative regulators of glucose-stimulated insulin secretion from pancreatic beta cells. Diabetologia. 2014;57(1):129-139. doi:10.1007/s00125-0133083-x

9. Christian P, Sacco J, Adeli K. Autophagy: emerging roles in lipid homeostasis and metabolic control. Biochim Biophys Acta. 2013;1831(4):819-824. doi:10.1016/j.bbalip.2012.12.009

10. Zamani M, Taher J, Adeli K. Complex role of autophagy in regulation of hepatic lipid and lipoprotein metabolism. J Biomed Res. 2017;31:377-385. doi:10.7555/JBR.30.20150137

11. Green DR, Levine B. To be or not to be? How selective autophagy and cell death govern cell fate. Cell. 2014;157(1):65-75. doi:10.1016/j.cell.2014.02.049

12. Li W, He P, Huang Y, et al. Selective autophagy of intracellular organelles: recent research advances. Theranostics. 2020;11:2 22-256. doi:10.7150/thno.49860

13. Zhang Z, Yao Z, Chen Y, et al. Lipophagy and liver disease: new perspectives to better understanding and therapy. Biomed Pharmacother. 2018;97:339-348. doi:10.1016/j.biopha.2017.07. 168

14. Singh R, Kaushik S, Wang Y, et al. Autophagy regulates lipid metabolism. Nature. 2009;458(7242):1131-1135. doi:10.1038/ nature 07976

15. Weidberg H, Shvets E, Elazar Z. Lipophagy: selective catabolism designed for lipids. Dev Cell. 2009;16(5):628-630. doi:10.1016/j. devcel.2009.05.001

16. Salimifar M, Fatehi-Hassanabad Z, Fatehi M. A review on natural products for controlling type 2 diabetes with an emphasis on their mechanisms of actions. Curr Diabetes Rev. 2013;9(5):402-411. doi:10.2174/15733998113099990076

17. Varshney R, Varshney R, Mishra R, et al. Kaempferol alleviates palmitic acid-induced lipid stores, endoplasmic reticulum stress and pancreatic $\beta$-cell dysfunction through AMPK/mTORmediated lipophagy. J Nutr Biochem. 2018;57:212-227. doi:10. 1016/j.jnutbio.2018.02.017

18. Sun Y, Xia M, Yan H, et al. Berberine attenuates hepatic steatosis and enhances energy expenditure in mice by inducing autophagy and fibroblast growth factor 21. Br J Pharmacol. 2018;175 (2):374-387. doi:10.1111/bph.14079

19. Ji J, Petropavlovskaia M, Khatchadourian A, et al. Type 2 diabetes is associated with suppression of autophagy and lipid accumulation in B-cells. J Cell Mol Med. 2019;23(4):2890-2900. doi: $10.1111 /$ jcmm.14172

20. Schofield JD, Liu Y, Rao-Balakrishna P, et al. Diabetes dyslipidemia. Diabetes Ther. 2016;7(2):203-219. doi:10.1007/ s13300-016-0167-x

21. Bano G. Glucose homeostasis, obesity and diabetes. Best Pract Res Clin Obstet Gynaecol. 2013;27(5):715-726. doi:10.1016/j. bpobgyn.2013.02.007

22. Virtue S, Vidal-Puig A. Adipose tissue expandability, lipotoxicity and the metabolic syndrome - an allostatic perspective. Biochim Biophys Acta. 2010;1801(3):338-349. doi:10.1016/j.bbalip.2009. 12.006

23. $\mathrm{Xu} \mathrm{S}$, Zhang X, Liu P. Lipid droplet proteins and metabolic diseases. Biochim Biophys Acta Mol Basis Dis. 2018;18 64:1968-1983

24. von Hanstein A-S, Lenzen S, Plötz T. Toxicity of fatty acid profiles of popular edible oils in human endoC- $\beta \mathrm{H} 1$ beta-cells Nutr Diabetes. 2020;10(1):1-5. doi:10.1038/s41387-020-0108-7
25. Quiclet C, Dittberner N, Gässler A, et al. Pancreatic adipocytes mediate hypersecretion of insulin in diabetes-susceptible mice. Metabolism. 2019;97:9-17. doi:10.1016/j.metabol.2019.05.005

26. Bays H, Mandarino L, DeFronzo RA. Role of the adipocyte, free fatty acids, and ectopic fat in pathogenesis of type 2 diabetes mellitus: peroxisomal proliferator-activated receptor agonists provide a rational therapeutic approach. J Clin Endocrinol Metab. 2004;89:463-478.

27. Khamzina L, Veilleux A, Bergeron S, et al. Increased activation of the mammalian target of rapamycin pathway in liver and skeletal muscle of obese rats: possible involvement in obesity-linked insulin resistance. Endocrinology. 2005;146 (3):1473-1481. doi:10.1210/en.2004-0921

28. Mottillo EP, Desjardins EM, Crane JD, et al. Lack of adipocyte AMPK exacerbates insulin resistance and hepatic steatosis through brown and beige adipose tissue function. Cell Metab. 2016;24(1):118-129. doi:10.1016/j.cmet.2016.06.006

29. Helene Knævelsrud H, Simonsen A. Lipids in autophagy: constituents, signaling molecules and cargo with relevance to disease. Biochim Biophys Acta. 2012;1821(8):1133-1145. doi:10.1016/j. bbalip.2012.01.001

30. Wang C-W. Lipid droplets, lipophagy, and beyond. Biochim Biophys Acta. 2016;1861(8):793-805. doi:10.1016/j.bbalip.20 15.12.010

31. Arcia EJ, Vevea JD, Pon LA. Lipid droplet autophagy during energy mobilization, lipid homeostasis and protein quality control. Front Biosci. 2018;23:1552-1563. doi:10.2741/4660

32. Liu K, Czaja MJ. Regulation of lipid stores and metabolism by lipophagy. Cell Death Differ. 2013;20:3-11. doi:10.1038/ cdd. 2012.63

33. Shin DW. Lipophagy: molecular mechanisms and implications in metabolic disorders. Mol Cells. 2020;43:686-693. doi:10.14348/ molcells.2020.0046

34. Zhang X, Evans TD, Jeong S-J, et al. Classical and alternative roles for autophagy in lipid metabolism. Curr Opin Lipidol. 2018;29(3):203-211. doi:10.1097/MOL.0000000000000509

35. Ward C, Martinez-Lopez N, Otten EG, et al. Autophagy, lipophagy and lysosomal lipid storage disorders. Biochim Biophys Acta. 2016;1861(4):269-284. doi:10.1016/j.bbalip.2016.01.006

36. Kaur J, Debnath J. Autophagy at the crossroads of catabolism and anabolism. Nat Rev Mol Cell Biol. 2015;16(8):461-472. doi: $10.1038 / \mathrm{nrm} 4024$

37. De Ballina LR, Munson MJ, Simonsen A. Lipids and lipid-binding proteins in selective autophagy. $J$ Mol Biol. 2019;1:135-159.

38. Gross AS, Graef M. Mechanisms of autophagy in metabolic stress response. J Mol Biol. 2020;432:28-52.

39. Papinski D, Kraft C. Regulation of autophagy by signaling through the Atg1/ULK1 Complex. J Mol Biol. 2016;428 (9):1725-1741. doi:10.1016/j.jmb.2016.03.030

40. Dalhaimer P. Lipid droplets in disease. Cells. 2019;8(9):974. doi:10.3390/cells 8090974

41. Rydén M, Andersson DP, Bergström IB, et al. Adipose tissue and metabolic alterations: regional differences in fat cell size and number matter, but differently: a cross-sectional study. J Clin Endocrinol Metab. 2014;99(10):E1870-E1876. doi:10.1210/ jc. 2014-1526

42. Morales PE, Bucarey JL, Espinosa A. Muscle lipid metabolism: role of lipid droplets and perilipins. $J$ Diabetes Res. 2017;2017:1789395. doi:10.1155/2017/1789395

43. Li X, Li Z, Zhao M, et al. Skeletal muscle lipid droplets and the athlete's paradox. Cells. 2019;8(3):249. doi:10.3390/cells8030249

44. Shen W, Gaskins HR, McIntosh MK. Influence of dietary fat on intestinal microbes, inflammation, barrier function and metabolic outcomes. J Nutr Biochem. 2014;25(3):270-280. doi:10.1016/j. jnutbio.2013.09.009 
45. Johnson AF, Olefsky JM. The origins and drivers of insulin resistance. Cell. 2013;152(4):673-684. doi:10.1016/j.cell.2013. 01.041

46. Antoni R, Johnston KL, Collins AL, et al. Caloric restriction and intermittent fasting alter hepatic lipid droplet proteome and diacylglycerol species and prevent diabetes in NZO mice. Biochim Biophys Acta. 2015;1851:566-576. doi:10.1016/j.bbalip.2015.01. 013

47. Antoni R, Johnston KL, Collins AL, et al. Effects of intermittent fasting on glucose and lipid metabolism. Proc Nutr Soc. 2017;76 (3):361-368. doi:10.1017/S0029665116002986

48. Al-mrabeh A, Zhyzhneuskaya SV, Peters C, et al. Hepatic lipoprotein export and remission of human type 2 diabetes after weight loss. Cell Metab. 2020;31(2):1-17. doi:10.1016/j. cmet.2019.11.018

49. Huang Q, Wang T, Yang L, et al. Ginsenoside Rb2 alleviates hepatic lipid accumulation by restoring autophagy via induction of sirt1 and activation of AMPK. Int J Mol Sci. 2017;18:1-15. doi:10.3390/ijms18051063

50. Chu KY, O'Reilly L, Ramm G, et al. High-fat diet increases autophagic flux in pancreatic beta cells in vivo and ex vivo in mice. Diabetologia. 2015;58(9):2074-2078. doi:10.1007/s00125015-3665-X

51. Zhang Y, Chen ML, Zhou Y, et al. Resveratrol improves hepatic steatosis by inducing autophagy through the cAMP signaling pathway. Mol Nutr Food Res. 2015;59:1443-1457. doi:10.1002/ mnfr.201500016

52. Zhu X, Xiong T, Liu P, et al. Quercetin ameliorates HFD-induced NAFLD by promoting hepatic VLDL assembly and lipophagy via the IRE1a/XBP1s pathway. Food Chem Toxicol. 2018;114:52-60. doi:10.1016/j.fct.2018.02.019

53. Zeng $\mathrm{H}$, Guo X, Zhou $\mathrm{F}$, et al. Quercetin alleviates ethanol-induced liver steatosis associated with improvement of lipophagy. Food Chem Toxicol. 2019;125:21-28. doi:10.1016/j. fct.2018.12.028

54. Pirillo A, Catapano AL. Berberine, a plant alkaloid with lipidand glucose-lowering properties: from in vitro evidence to clinical studies. Atherosclerosis. 2015;243:449-461. doi:10.1016/j. atherosclerosis.2015.09.032

55. Zhu X, Yang L, Xu F, et al. Combination therapy with catechins and caffeine inhibits fat accumulation in 3T3-L1 cells. Exp Ther Med. 2017;13:688-694. doi:10.3892/etm.2016.3975

56. Sinha RA, Farah BL, Singh BK, et al. Caffeine stimulates hepatic lipid metabolism by the autophagy-lysosomal pathway in mice. Hepatology. 2014;59:1366-1380. doi:10.1002/hep.26667

57. Li Z, Ji GE. Ginseng and obesity. J Ginseng Res. 2018;42(1):1-8. doi:10.1016/j.jgr.2016.12.005

58. Kounakis K, Chaniotakis M, Markaki M, et al. Emerging roles of lipophagy in health and disease. Front Cell Dev Biol. 2019;7:185. doi:10.3389/fcell.2019.00185

59. Schulze RJ, Drižytė K, Casey CA, McNiven MA. Hepatic lipophagy: new insights into autophagic catabolism of lipid droplets in the liver. Hepatol Commun. 2017;1(5):359-369. doi:10.1002/hep4.1056

60. Lee H, Lee H, Lim Y. Vitamin D3 improves lipophagy-associated renal lipid metabolism and tissue damage in diabetic mice. Nutr Res. 2020;80:55-65. doi:10.1016/j.nutres.2020.06.007

61. Davidson MB, Peters AL. An overview of metformin in the treatment of type 2 diabetes mellitus. Am J Med. 1997;102 (1):99-110. doi:10.1016/S0002-9343(96)00353-1

62. Lettieri Barbato D, Tatulli G, Aquilano K, et al. FoxO1 controls lysosomal acid lipase in adipocytes: implication of lipophagy during nutrient restriction and metformin treatment. Cell Death Dis. 2013;4(10):1-11. doi:10.1038/cddis.2013.404

63. Hur KY, Lee M-S. New mechanisms of metformin action: focusing on mitochondria and the gut. J Diabetes Investig. 2015;6 (6):600-609. doi:10.1111/jdi.12328
64. Bai L, Gao J, Wei F, et al. Therapeutic potential of ginsenosides as an adjuvant treatment for diabetes. Front Pharmacol. 2018;9:423. doi:10.3389/fphar.2018.00423

65. Meng F, Ning H, Sun Z, et al. Ursolic acid protects hepatocytes against lipotoxicity through activating autophagy via an AMPK pathway. $J$ Funct Foods. 2015;17:172-182. doi:10.1016/j. jff.2015.05.029

66. Xia E-Q, Yu -Y-Y, Xu X-R, et al. Ultrasound-assisted extraction of oleanolic acid and ursolic acid from ligustrum lucidum ait. Ultrason Sonochem. 2012;19(4):772-776. doi:10.1016/j.ultsonch. 2011.11.014

67. Lee DH, Park SH, Huh YH, et al. Iridoids of valeriana fauriei contribute to alleviating hepatic steatosis in obese mice by lipophagy. Biomed Pharmacother. 2020;125:109950. doi:10.10 16/j.biopha.2020.109950

68. Jin Y, Khadka DB, Cho WJ. Pharmacological effects of berberine and its derivatives: a patent update. Expert Opin Ther Pat. 2016;26:229-243. doi:10.1517/13543776.2016.1118060

69. Calderón-Montaño JM, Burgos-Morón E, Pérez-Guerrero C, et al. A review on the dietary flavonoid kaempferol. Mini Rev Med Chem. 2011;11(4):298-344. doi:10.2174/138955711795305335

70. Wang Y, Zhao H, Li X, et al. Formononetin alleviates hepatic steatosis by facilitating TFEB-mediated lysosome biogenesis and lipophagy. J Nutr Biochem. 2019;73:108214. doi:10.1016/j. jnutbio.2019.07.005

71. Jiang D, Rasul A, Batool R, et al. Potential anticancer properties and mechanisms of action of formononetin. Biomed Res Int. 2019;2019:5854315. doi:10.1155/2019/5854315

72. Haga S, Yamaki H, Jin S, et al. Extracts of bilberry (Vaccinium myrtillus L.) fruits improve liver steatosis and injury in mice by preventing lipid accumulation and cell death. Biosci Biotechnol Biochem. 2019;83(11):2110-2120. doi:10.1080/09168451.2019. 1634514

73. D'Andrea G. Quercetin: a flavonol with multifaceted therapeutic applications? Fitoterapia. 2015;106:256-271. doi:10.1016/j. fitote.2015.09.018

74. Pezzuto JM. Resveratrol: twenty years of growth, development and controversy. Biomol Ther. 2019;27(1):1-14. doi:10.4062/ biomolther.2018.176

75. Zhou J, Farah BL, Sinha RA, et al. Epigallocatechin-3-gallate (EGCG), a green tea polyphenol, stimulates hepatic autophagy and lipid clearance. PLoS One. 2014;9(1):e87161. doi:10.1371/ journal.pone.0087161

76. Parafati M, Lascala A, Morittu VM, et al. Bergamot polyphenol fraction prevents nonalcoholic fatty liver disease via stimulation of lipophagy in cafeteria diet-induced rat model of metabolic syndrome. J Nutr Biochem. 2015;26(9):938-948. doi:10.1016/j. jnutbio.2015.03.008

77. Liu M, Xu L, Yin L, et al. Potent effects of dioscin against obesity in mice. Sci Rep. 2015;5(1):7973. doi:10.1038/srep07973

78. Zhang Y, Jin L, Liu J, et al. Effect and mechanism of dioscin from Dioscorea spongiosa on uric acid excretion in animal model of hyperuricemia. J Ethnopharmacol. 2018;214:29-36. doi:10.1016/ j.jep.2017.12.004

79. DeBosch BJ, Heitmeier MR, Mayer AL, et al. Trehalose inhibits solute carrier 2A (SLC2A) proteins to induce autophagy and prevent hepatic steatosis. Sci Signal. 2016;9(416):ra21. doi:10. 1126/scisignal.aac5472

80. Janda E, Lascala A, Martino C, et al. Molecular mechanisms of lipidand glucose-lowering activities of bergamot flavonoids. PharmaNutrition. 2016;4:S8-S18. doi:10.1016/j.phanu.2016.05.001

81. Xu L, Li Y, Dai Y, Peng J. Natural products for the treatment of type 2 diabetes mellitus: pharmacology and mechanisms. Pharmacol Res. 2018;130:451-465. doi:10.1016/j.phrs.2018. 01.015 
82. Cheng $\mathrm{C}, \mathrm{Li} \mathrm{Z}$, Zhao $\mathrm{X}$, et al. Natural alkaloid and polyphenol compounds targeting lipid metabolism: treatment implications in metabolic diseases. Eur J Pharmacol. 2020;870:172922. doi:10. 1016/j.ejphar.2020.172922

83. Castellano JM, Espinosa JM, Perona JS. Modulation of lipid transport and adipose tissue deposition by small lipophilic compounds. Front Cell Dev Biol. 2020;8:1-15. doi:10.3389/fcell.2020.555359

84. Farese RV, Walther TC. Lipid droplets finally get a little R-E-S-P-E-C-T. Cell. 2009;139(5):855-860. doi:10.1016/j.cell. 2009.11.005

85. Hussain MM. Intestinal lipid absorption and lipoprotein formation. Curr Opin Lipidol. 2014;25(3):200-206. doi:10.1097/ MOL.0000000000000084

86. Kassan A, Herms A, Fernández-Vidal A, et al. Acyl-CoA synthetase 3 promotes lipid droplet biogenesis in ER microdomains. J Cell Biol. 2013;203(6):985-1001. doi:10.1083/jcb.201305142

87. Karpe F, Dickmann JR, Frayn KN. Fatty acids, obesity, and insulin resistance: time for a reevaluation. Diabetes. 2011;60 (10):2441-2449. doi:10.2337/db11-0425

88. Zhou K, Yao P, He J, et al. Lipophagy in nonliver tissues and some related diseases: pathogenic and therapeutic implications. J Cell Physiol. 2019;234(6):7938-7947. doi:10.1002/jcp.27988

89. Zechner R, Madeo F, Kratky D. Cytosolic lipolysis and lipophagy: two sides of the same coin. Nat Rev Mol Cell Biol. 2017;18(11):671-684. doi:10.1038/nrm.2017.76

90. Kloska A, Węsierska M, Malinowska M, et al. Lipophagy and lipolysis status in lipid storage and lipid metabolism diseases. Int J Mol Sci. 2020;21(17):6113. doi:10.3390/ijms21176113

91. Jaishy B, Abel ED. Lipids, lysosomes, and autophagy. J Lipid Res. 2016;57(9):1619-1635. doi:10.1194/j1r.R067520

92. Lizaso A, Tan K-T, Lee Y-H. $\beta$-adrenergic receptor-stimulated lipolysis requires the RAB7-mediated autolysosomal lipid degradation. Autophagy. 2013;9(8):1228-1243. doi:10.4161/auto.24893

93. Zhang Z, Zhao S, Yao Z, et al. Autophagy regulates turnover of lipid droplets via ROS-dependent Rab25 activation in hepatic stellate cell. Redox Biol. 2017;11:322-334. doi:10.1016/j.redox. 2016.12.021

94. Settembre C, De Cegli R, Mansueto G, et al. TFEB controls cellular lipid metabolism through a starvation-induced autoregulatory loop. Nat Cell Biol. 2013;15(6):647-658. doi:10.1038/ ncb2718
95. Bugliani M, Mossuto S, Grano F, et al. Modulation of autophagy influences the function and survival of human pancreatic beta cells under endoplasmic reticulum stress conditions and in type 2 diabetes. Front Endocrinol. 2019;10:1-10. doi:10.3389/ fendo.2019.00052

96. Stienstra R, Haim Y, Riahi Y, et al. Autophagy in adipose tissue and the beta cell: implications for obesity and diabetes. Diabetologia. 2014;57(8):1505-1516. doi:10.1007/s00125-0143255-3

97. Bachar-Wikstrom E, Wikstrom JD, Kaiser N, et al. Improvement of ER stress-induced diabetes by stimulating autophagy. Autophagy. 2013;9(4):626-628. doi:10.4161/auto.23642

98. Gonzalez CD, Lee M-S, Marchetti P, et al. The emerging role of autophagy in the pathophysiology of diabetes mellitus. Autophagy. 2011;7(1):2-11. doi:10.4161/auto.7.1.13044

99. Flamment M, Hajduch E, Ferré P, et al. New insights into ER stress-induced insulin resistance. Trends Endocrinol Metab. 2012;23(8):381-390. doi:10.1016/j.tem.2012.06.003

100. Quan W, Lim YM, Lee MS. Role of autophagy in diabetes and endoplasmic reticulum stress of pancreatic $\beta$-cells. Exp Mol Med. 2012;44:81-88. doi:10.3858/emm.2012.44.2.030

101. Yang L, Li P, Fu S, et al. Defective hepatic autophagy in obesity promotes ER stress and causes insulin resistance. Cell Metab. 2010;11(6):467-478. doi:10.1016/j.cmet.2010.04.005

102. Gao Y, Zhang W, Zeng LQ, et al. Exercise and dietary intervention ameliorate high-fat diet-induced NAFLD and liver aging by inducing lipophagy. Redox Biol. 2020;36:101635. doi:10.1016/j. redox.2020.101635

103. Merlotti C, Ceriani V, Morabito A, et al. Subcutaneous fat loss is greater than visceral fat loss with diet and exercise, weight-loss promoting drugs and bariatric surgery: a critical review and meta-analysis. Int $J$ Obes. 2017;41(5):672-682. doi:10.1038/ ijo. 2017.31

\section{Publish your work in this journal}

Diabetes, Metabolic Syndrome and Obesity: Targets and Therapy is an international, peer-reviewed open-access journal committed to the rapid publication of the latest laboratory and clinical findings in the fields of diabetes, metabolic syndrome and obesity research. Original research, review, case reports, hypothesis formation, expert opinion and commentaries are all considered for publication. The manuscript management system is completely online and includes a very quick and fair peer-review system, which is all easy to use. Visit http://www.dovepress.com/testimonials.php to read real quotes from published authors. 